\title{
The Effect of Gluteus Medius Training on Hip Kinematics in a Runner with Iliotibial Band Syndrome
}

\begin{abstract}
Iliotibial band syndrome (ITBS) is a common clinical presentation in runners. There are several hypotheses to explain this condition including faulty control of the hip joint in the frontal plane during the stance phase of running. It is postulated that improving activity in the gluteus medius muscle may assist in producing more appropriate stabilization and therefore reduce the stress on the Iliotibial band (ITB). This single case study provides an interesting clinical scenario where a single-subject with ITBS was measured for hip kinematics during running, before and after a trial period of classic gluteus medius exercises. The biomechanical data show an initial (pre-intervention) increase in adduction position during the stance phase of running on the affected side (in contrast to the unaffected side). This was measured using a MOVEN motion analysis suit. After the trial intervention period, the relative position of the affected hip had reduced in adduction at both heel strike and at $30^{\circ}$ knee flexion. This study provides support for the theory that hip control in the frontal plane may be a contributing factor in ITBS. Clinicians are encouraged to monitor hip control as well as ITBS symptoms when they utilise this gluteus medius protocol. Further research to establish whether change in pelvic control results in decrease in ITBS symptoms is warranted.
\end{abstract}

KEY WORDS: ILIOTIBIAL BAND SYNDROME, RUNNERS, HIP KINEMATICS, BIOMECHANICS, MOTION ANALYSIS.

\section{BACKGROUND}

Iliotibial band syndrome (ITBS) is the second leading cause of knee pain and the primary cause of lateral knee pain in runners (Noehren et al 2007). ITBS makes up $12 \%$ of all overuse injuries in running and $22 \%$ of all lower extremity running injuries (Fredericson and Weir 2006, Grau et al 2008, Taunton et al 2002). Due to the increased awareness of aerobic exercise to maintain a healthy lifestyle, jogging and running have become more popular. This is mirrored by an increase in acute and chronic running injuries (Dugan and Bhat 2005). ITBS is common among cyclists, football players, hockey players, downhill skiers, weight lifters and long distance runners (Gunter and Schwellnus 2004).

In long-distance running, repetitive flexion and extension of the knee joint leads to excessive rubbing of the distal iliotibial band across the lateral femoral condyle (Grau et al 2008). The pain usually increases over the duration of the run due to cumulative effects of the repetitive knee flexion/ extension movements and consequently leads to an inflammatory process (Franco et al 1997, Fredericson and Wolf 2006, Khaund and Flynn 2005). In severe cases, the runner may not be able to continue running and needs to seek medical attention (Noehren et al 2007). ITBS in runners is thus a significant problem, as the intensity of the pain can lead to attrition from running (Miller et al 2007).

Pain develops after heel strike in the initial standing phase, while the knee joint angle is in less than 30 degrees of flexion (Fredericson and Wolf 2005). This may be the maximal zone of impingement during running (Fredericson and Weir 2006). A relative smaller knee flexion angle at heel strike predisposes a runner to ITBS, since more time is then spent in the impingement arc of knee flexion (Fredericson and Wolf 2005). The relatively smaller knee flexion angle has been suggested to be due to hip muscle weakness of the gluteus medius (Fredericson et al 2000). However it is not entirely clear why distance runners may be prone to this weakness. Fredericson et al (2000) proposed that running is mainly a sagittal plane activity which requires less hip control in the frontal plane.

Biomechanical analysis can unlock explanations to presentations such as ITBS, but there is a dearth of biomechanical research into understanding the relationship between hip control and ITBS in runners (Fairclough et al 2007). In healthy runners, the gluteus medius, especially the posterior part, is primarily a global stabilizer which initiates external rotation of the hip. In addition, the gluteus medius muscle should contract eccentrically at heel strike and then concentrically during the stance

\section{Corresponding Author:}

Prof Quinette Louw

Physiotherapy Division

Stellenbosch University

Fransie van Zijl Avenue

Parow Valley 7500

E-mail: qalouw@sun.ac.za 
phase to control frontal plane hip movements (Fredericson et al 2000). Although the role of the gluteus medius during the running cycle in healthy individuals has been established, the role of this muscle in ITBS remains vague.

A recent ITBS biomechanical theory is that the degree of frontal plane hip angles may influence an individual's risk of developing ITBS (Miller et al 2008). During running, increased hip adduction and internal rotation angles during the stance phase may increase strain on the ITB (Fredericson and Wolf 2005, Noehren et al 2007). Miller et al (2008) further reported that runners who were prone to develop ITBS demonstrated coupled hip adduction/abduction and tibia internal/external rotation movements. These coupled knee patterns may increase tension on the ITB, with ensuing micro-trauma of the tissue (Fredericson et al 2000). These coupled movements may therefore be an effect or cause of ITBS (Noehren et al 2007).

Gluteus medius muscle weakness may be related to impaired hip frontal plane control (Fredericson et al 2000). Weakness of this muscle may lead to relatively increased hip adduction and internal rotation and an increased valgus vector at the knee (Fredericson et al 2000, Niemuth et al 2005). This finding is in agreement with researchers who postulate that impaired gluteus medius control is a predisposing factor for ITBS (Fairclough et al 2007). Grau et al (2008) reported no difference in hip abduction muscle strength between runners with and without ITBS, but a shortcoming was that functional biomechanical analyses of hip control during running was not conducted. The importance of the hip musculature in the prevention and treatment of ITBS thus remains controversial. The aim of this study was thus to ascertain the effect of a gluteus medius strengthening program on hip control during the stance phase of running in a runner with ITBS.

\section{METHODOLOGY}

This study was approved by the Human Research Ethics Committee of the Faculty of Health Sciences, Stellenbosch University (N08/09/245) and the subject provided signed consent. Permission to conduct the study was obtained from the Performance Institute of Stellenbosch University (SUSPI) where the study was conducted.

\section{Participant recruitment and eligibility criteria}

The participant was recruited from the physiotherapy clinic of SUSPI. The inclusion criteria were: an amateur, male or female runner that ran on average between 80 and $100 \mathrm{~km}$ per week and competed in races without financial incentives. The participant was recruited being positive for the following criteria: Older than 18 years with complaints of lateral knee pain during or after running and which increased with downhill running; positive testing with the Ober, Noble and hop tests as well as on palpation of the ITB over the lateral condyle of the femur; wearing neutral running shoes without orthotics; good general health, no associated musculoskeletal injuries, and should not have been receiving any other form of medical or alternative treatment. An objective inclusion criterion was relative weakness of the gluteus medius muscle on the affected side compared with the unaffected side as measured by a hand-held dynamometer. Relative weakness was defined as at least $20 \%$ less strength compared to the unaffected side (Maffiuletti 2010). The exclusion criteria were previous lower limb injuries or any soft tissue treatment received for current ITBS.

\section{Study design}

A single-subject, ABA -design study (where A represents the baseline phases and $\mathrm{B}$ the treatment phase) was conducted over eight weeks. The study timeline consisted of the following three phases:

Baseline phase (A1): Baseline assessments were conducted one week after the inclusive criteria were met and the participant had been diagnosed with ITBS. The assessments included biomechanical analyses of the hip joint angles in the frontal plane (hip adduction/ adduction) on three alternate days in the same week (Monday, Wednesday and Friday). Hip angles were analysed using the MOVEN motion analysis system. Pain was monitored using the Visual Analogue Scale (VAS) on all three days when the biomechanical analyses were done.

Intervention phase (B): During this phase, the six-week gluteus medius strengthening program was implemented.

The follow-up phase (A2): Biomechanical analyses were of the frontal hip joint angles were repeated on three alternate days during the eighth week of the study. Pain was monitored using the VAS.

\section{Intervention}

The intervention was a six-week, daily gluteus medius strengthening program which was aimed at increasing muscle control and local muscle endurance on the affected leg. The mode of delivery was a combination of supervised and home-based sessions. Three supervised sessions which lasted about 40 minutes were conducted at SUSPI throughout the eight weeks intervention phase. The principle researcher supervised all sessions. In addition, the participant received a printed copy of the program at the beginning of the intervention program to continue the exercises at home, bar the days when attending sessions at SUSPI. The home-based exercises were followed up telephonically.

\section{Description of exercises:}

The program consisted of the exercises described in figures 1, 2 and 3. The rationale of including transverse abdominal muscle exercises is that function of this stabilizer is a prerequisite to any form of strength training as it ensures load balance within the kinetic chain (Akuthota et al 2008). Core stability is imperative for initiation of functional limb movements (Akuthota et al 2008). In this case, the activation of transverse abdominals was important to keep the spine in the neutral position during execution of the hip exercises. Electromyographic studies have illustrated that the exercises in figures 2 and 3 are optimal for improving strength control of the gluteus medius (Distefano et al 2009). 


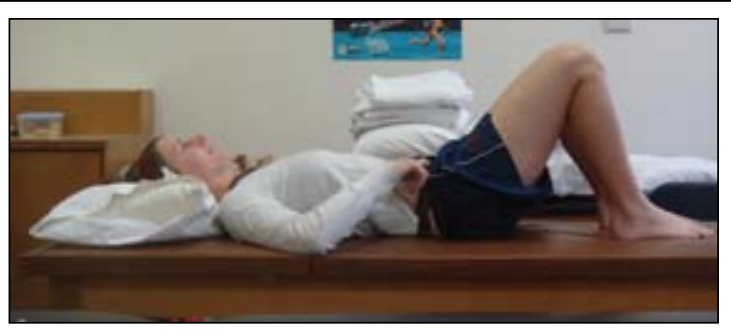

Figure 1: Transverse Abdominal activation. Hold the position and do 8 breaths 10 times (Hodges and Richardson 1996)

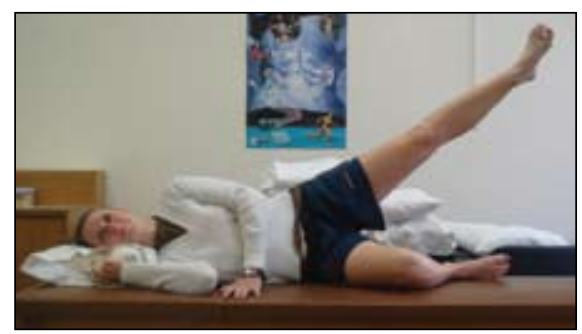

Figure 2 A

Figure 2 A and B: A) Clam - Beginner phase, transverse abdominals activated, hold the position and lift the knee. Repeat 8 times, three sets. B) Straight leg raise in side lying (progression of Clam). Side lying with transverse abdominal activated. Repeat 8 times four sets (Fredericson et al 2000).
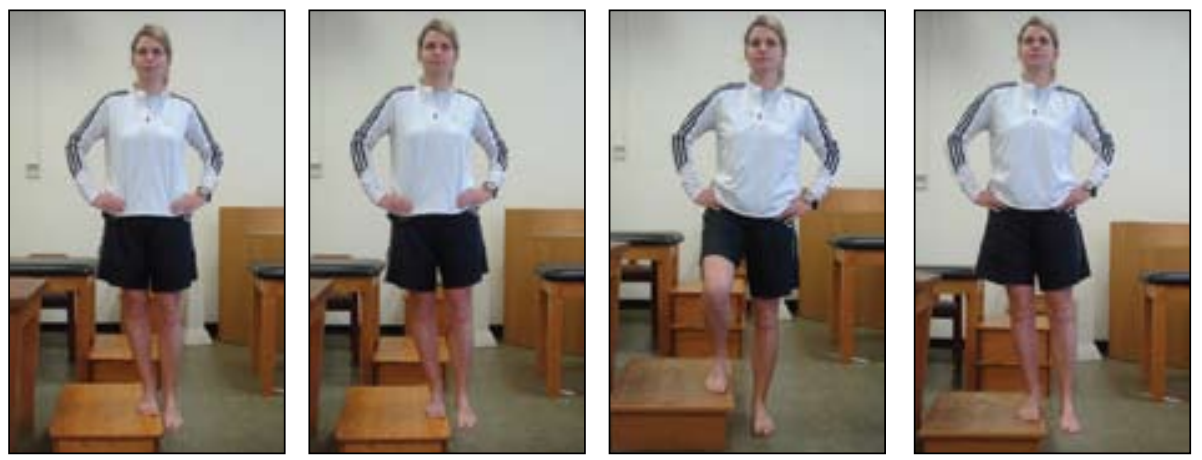

Figure 3 AB

Figure 3 A and B: A) Single Leg Hitch of Step - Spine in neutral with transverse abdominus activated. The gluteus medius of the affected side slowly pulls the unaffected pelvis upwards to neutral spine. Repeat 8 times, three sets. (Fredericson and Weir 2006, Fredericson et al 2000). B) Sideways step up (Progression of Single Leg Hitch). Side step up with transverse abdominal activated. Repeat 8 times, four sets (Fredericson and Wolf 2005, Khaund and Flynn, 2005).

\section{Verbal feedback during exercise execution}

The exercises were monitored and corrected as necessary by the principle researcher. If pain was experienced in the knee, the exercise technique was corrected by verbal feedback. Pain may be an indication that strain is imposed on the ITB that may lead to increased hip internal rotation and consequent irritation over the distal part of ITB.

\section{Study Procedures}

\section{Clinical evaluation}

\section{- ITBS diagnostic tests}

At the first appointment (day one), a subjective and physical examination was conducted using clinical diagnostic tests including the Noble test, the Hop Test, the Ober test and palpation over the distal ITB area (Barber and Sutker 1992, Franco et al 1997, Fredericson and Weir 2006, Khaund and Flynn 2005). The participant was diagnosed with ITBS because both the Noble and Hop tests and the palpation were positive, and subjectively reported lateral knee pain while or after running (Fredericson and Weir 2006).

- Gluteus medius muscle strength test In side-lying, the participant produced an isometric action of hip abduction for three to five seconds in approximately $30^{\circ}$ of hip abduction and $5^{\circ}$ of hip extension with the hip in neutral rotation (Fredericson et al, 2000). Maximum resistance was applied by the researcher using a hand-held dynamometer proximal to the lateral malleolus (Fredericson et al, 2000). During the testing, continuous monitoring of movement was done to ensure appropriate stabilisation and that no hip flexion was allowed and rest periods were given between testing. During the test procedures of the gluteus medius strength, no pain was produced at any stage. Three measurements were taken and the average score was used for the final score.

The muscle strength test using the hand-held dynamometer has demonstrated good test-retest reliability and the validity assessments have been compared to isokinetic testing of shoulder and hamstrings (Hyde et al, 1983, Bohannon et al, 1986). We need to acknowledge that with the strength test of gluteus medius the tensor fasia latae and gluteus minimus could have been recruited due to both being hip abductors (Hollingshead et al 1982). 


\section{- Pain assessment}

The principal researcher administered a uni-dimensional Visual Analogue scale (VAS) for resting pain as well as pain with walking and running (Collins et al, 1997).

\section{Motion analysis of hip angles during running}

The biomechanical measurement of hip motion in the frontal plane was done using the MOVEN motion analysis system (developed by Xsens Technology B.V, Netherlands, inertial motion system). The MOVEN motion analysis system consists of Lycra strips with sixteen attached inertial sensors which are placed over the joints. Each sensor is comprised of a 3D gyroscope, 3D accelerometers and 3D magnetometers. These motion trackers give the total orientation estimate of the hip. This was used to calculate the $3 \mathrm{D}$ linear acceleration, giving the translation estimate of the joint. All the sensors were positioned to ensure accurate orientation of the suit, although only hip angles were analysed. Validity and reliability of the MOVEN, compared to the Vicon motion analysis system have been previously established by Scheffer and Cloete (2008).

The biomechanical analysis was conducted in the Stellenbosch University Gymnasium Sports Hall. The participant started with a two-minute, warm-up cycle before running. No pain was present before any biomechanical testing was conducted or during the running trails. The participant was then instructed to run at a speed of $9-10 \mathrm{~km} /$ hour, which is reportedly the participant's average running speed when training. The speed was monitored with the MOVEN's detachable speedometer.

One running trial of ten minutes was captured on each of the three alternate test days during the baseline and followup phases. The last two minutes of the ten-minute run were captured, however the participant was blinded as to the length of the run and to when the data were recorded, hence he altered his style. The biomechanical data of the last three steps of the tenth minute during the run was analysed for each of the three trials (one trial on three alternate days) Therefore, the hip biomechanics of a total of nine steps were analysed for the baseline and the follow-up phases. Reliability and repeatability for the biomechanical assessment was conducted on the same day before the trails were performed.

The data were transferred wirelessly to a laptop to process the hip kinematics. The motion capture's software converts acceleration and orientation measurements (obtain form motion capture sensors) to translation (three Cartesian components) and rotation (quaternion vector) measurements. The orientation of each segment is calculated by means of quaternion multiplication (Baker 2009). This enables the calculation of each joint's flexion angle by subtracting the orientation measures of the joint's inner and outer segment. Each joint's translation in 3-D space and its rotation angles are therefore available for export, in this case, an excel spreadsheet. The point of heel strike and $30^{\circ}$ was visually estimated by the engineer. The data were then accurately documented and organised in columns which included the day, step number, time frame and abduction in an Excel spreadsheet for each leg.

\section{Data Analysis}

Descriptive statistical techniques were used to analyse the hip adduction data. The measurement closest to heel strike and to $30^{\circ}$ of knee flexion of each of the three steps in the trial was documented and plotted in an MSExcel spread sheet. The definition of direction of movement was stipulated by the engineer and the MOVEN analysis system. The abduction movement in the affected (right) leg was negative and adduction was positive. In the unaffected leg (left) it was the reverse i.e. adduction was negative and abduction was positive.

\section{RESULTS}

\section{Subject description}

The participant was a 21 year-old male, who had no history of any injury to his affected leg which was his right (and dominant) leg. His running program consisted of 15 to $20 \mathrm{~km}$ per day on the road during the week and $30 \mathrm{~km}$ 's on a Saturday. Sundays were rest days. $\mathrm{He}$ consistently ran on the same surfaces, and did not change any of his training intensity or running shoes. His shoes were deemed to be in good condition and appropriate for the study. Mean gluteus medius strength of the affected leg was $12.5 \mathrm{~kg}$ (SD: 1.95) and the unaffected

Table 1: Means (sd) of pre- and post-test scores for the position of the affected and unaffected hips at two points in the running cycle, measured in degrees.

\begin{tabular}{|l|l|l|l|l|}
\hline & $\begin{array}{l}\text { Affected leg at } \\
\text { heel strike }\end{array}$ & $\begin{array}{l}\text { Unaffected leg at } \\
\text { heel strike }\end{array}$ & $\begin{array}{l}\text { Affected leg at } \mathbf{3 0}^{\circ} \\
\text { knee flexion }\end{array}$ & $\begin{array}{l}\text { Unaffected leg at } \mathbf{3 0}^{\circ} \\
\text { knee flexion }\end{array}$ \\
\hline $\begin{array}{l}\text { Pre- intervention } \\
\text { Mean(sd) }\end{array}$ & $+3.27(2.76)$ add & $+0.58(6.43) \mathrm{abd}$ & $+12.4(2.68)$ add & $-5.64(4.00)$ add \\
\hline $\begin{array}{l}\text { Post- intervention } \\
\text { Mean(sd) }\end{array}$ & $-0.20(4.14)$ abd & $-3.7(4.29)$ add & $+9.8(3.37)$ add & $-12.1(2.4)$ add \\
\hline $\begin{array}{l}\text { Change score } \\
\text { Mean (sd) }\end{array}$ & $-3.47(5.74)$ & $-0.78(7.22)$ & $-2.63(3.86)$ & $-6.46(6.15)$ \\
\hline
\end{tabular}

NB - affected leg abduction values $=-$ ve, adduction values $=+$ ve; non-affected abduction values $=+$ ve, adduction values $=-$ ve 
leg was $20 \mathrm{~kg}$ (SD: 2.59). Thus the affected side was $37.5 \%$ weaker than the unaffected side.

The pre-intervention biomechanical data showed an increase in hip adduction of the affected leg (M: $3.27^{\circ}$, SD: 2.76) compared to the unaffected side at heel strike (M: $\left.0.58^{\circ} \mathrm{SD} 6.43\right)$ and at $30^{\circ}$ knee flexion (affected M: $12.4^{\circ}$ adduction, SD: 2.68 versus unaffected M: $5.64^{\circ}$, SD: 4.00) (see Table 1).

After the intervention phase, the affected leg had a mean decrease in hip adduction at the affected leg at heel strike (M: $0.20^{\circ}$ abduction, SD: 4.14) and at $30^{\circ}$ knee flexion (M: $9.80^{\circ}$ adduction, SD: 3.37) (see table 1). After the intervention phase there was an increase in adduction of the unaffected leg at heel strike and at $30^{\circ}$ of knee flexion.

\section{Pain VAS assessment}

The participant reported a 5/10 VAS pain score with walking and jogging and no pain at rest at the first day of assessment. A week later, the participant reported a VAS of ' 0 ' with walking or any activities, indicated that no pain was experienced before the study was conducted. All subsequent pain measures were $0 / 10$ VAS during the running trails of this study.

\section{DISCUSSION}

The main objective of this study was to investigate the biomechanical effect of gluteus medius training (which focused on the control and endurance in the frontal plane of hip kinematics) during the stance phase of running in a patient with ITBS.

Gluteus medius strengthening has been part of a wide range of treatment plans for athletes with ITBS. Other interventions previously reported for ITBS include stretching, soft tissue mobilization, dry needling and biomechanical correction of the footwear (Fredericson and Weir 2005).

The biomechanical test results of this study during the pre-intervention phase reported increased hip adduction in the affected leg, with the gluteus medius weakness compared to the unaffected leg. This confirmed that the participant in this study presented with a similar clinical presentation to those described in the biomechanical study of Noehren et al (2007). This adds further support that a relationship exists between the hip muscle weakness and an injury pattern in runners suffering from ITBS.

With the use of the MOVEN system, measurements after the intervention stage reported a reduction of adduction at heel strike and $30^{\circ}$ knee flexion of the affected side during running (mean change $3.47^{\circ}$ and $2.63^{\circ}$ respectively). According to Scheffer and Cloete (2008) any change result of two degrees or less indicates an inaccurate measurement or no change. Therefore we have evidence to suggest that there was a true change in hip abduction control during the study period. These changes in kinematics observed might have been clinically relevant even bearing in mind the large standard deviations indicate quite large variance. Given the study design we cannot say with certainty that the change was as a result of the intervention.

The uninvolved leg was also measured and monitored by the MOVEN system before and after the intervention. Before the intervention, the unaffected leg with a $37.5 \%$ stronger gluteus medius strength reported an increased in hip abduction compared to the affected hip. The intervention phase was only conducted on the gluteus medius of the affected side. Although at the end of the postintervention phase the unaffected leg reported a decrease in abduction of the hip. This could be due to compensatory strategies of the pelvis for stability after only training a specific muscle group. Currently, no published evidence could be found to support this.

There were many limitations to this study. Although the MOVEN system showed reliability according to Scheffer and Cloete (2008), the sensitivity of the system at heel strike point was questionable due to the subjective time-point estimate of the engineer. Furthermore, limited conclusions can be made from a single-case design. A larger sample size will be of more value to confirm this study's preliminary findings. Measuring post-intervention strength of gluteus medius would have indicated that a six-week program of the above- mentioned exercises was either adequate or inadequate for an athlete to increase in strength and endurance. The participant did continue with cycling for $30 \mathrm{~min}$ utes, on alternative days, to keep fit. $\mathrm{He}$ was cycling at the same intensity before the study, so this probably did not affect the end result of the study. The participant complied with all required exercises and stayed within the parameters of the study.

A longer trial period may be needed to measure gluteus medius strength/ control because it is a global stabilizer that may need more than a six-week strength training program (Hodges and Richardson 1996). The exercises could have also been progressed with adding resistance to increase the level of muscle activation and potentially improve the strengthening effects. It must be remembered, though, that it is not necessarily strength that improves functional ability, but rather motor coordination parameters such as timing, sequencing or grading of force production.

\section{CONCLUSION}

The high incidence numbers of ITBS among long-distance runners demonstrates the importance of more biomechanical research in athletes with ITBS (Noehren et al 2007). The use of the gluteus medius strengthening program which focuses on endurance and control demonstrated changes in the abduction/adduction hip angles during the stance phase of running. Clinicians should continue to trial this intervention with clear outcome measures to monitor individual responses in ITBS symptoms. In future studies, larger numbers should be included to further our understanding of the faulty kinematics in ITBS during running. 


\section{REFERENCES}

Akuthota V, Ferreiro A, Moore T, Fredericson M 2008 Core Stability exercise principles. Current Sports Medical Report 7:39-44

Baker M 2009 Maths - Transformation using Quaternions [Online] [Accessed 13 October 2009]. Available: http://www.euclideanspace.com/maths/ algebra/realNormedAlgebra/quaternions/transforms/index.htm>

Barber A, Sutker A 1992 Iliotibial Band Syndrome. Sports Medicine 14:144-148

Bohannon RW 1982 Test-retest reliability of hand-held dynamometry during a single session of strength assessment. Physical Therapy 66: 206-209

Collins SL, Moore RA, Mcquay HJ 1997 The visual analogue pain intensity scale: what is moderate pain in millimetres?. Pain 72: 95-97

Distefano L, Blackburn J, Marshall S, Padua D 2009 Gluteal Muscle Activation During Common Therapeutic Exercises. Journal of Orthopaedic and Sports Physical Therapy 7:532-540

Dugan S, Bhat K 2005 Biomechanics and Analysis of Running Gait. Physical Medicine Rehabilitation Clinics of North America 16: 603-621

Fairclough J, Hayashi K, Toumi H, Lyons K, Bydder G, Phillips N, Best T, Benjamin M 2007 Is iliotibial band syndrome really a friction syndrome? Journal of Science and Medicine in Sport 10:74-76

Franco V, Cerullo G Gianni E, Puddu G 1997 Iliotibial Band Friction Syndrome. Operative Techniques in Sports Medicine 5:153-156

Fredericson M, Weir A 2006 Practical Management of Iliotibial band friction syndrome in runners. Clinical Journal of Sport Medicine $16: 261-268$

Fredericson M, Wolf C 2005 Iliotibial band syndrome in runners: innovations in treatment. Sports Medicine 35:451-459

Fredericson M, Cookingham C, Chaudhari A, Dowdell B, Oestreicher N, Sahrmann A 2000 Hip Abductor weakness in Distance Runners with Iliotibial Band Syndrome. Clinical Journal of Sport Medicine 10:169-175
Grau S, Maiwald C, Krauss I, Axmann D, Horstmann T 2008 The Influence of Matching Populations on Kinematic and Kinetic Variables in Runners with Iliotibial Band Syndrome. Research Quaterly for Exercise and Sport 79:450-457

Gunter P, Schwellnus M 2003 Local corticosteroid injection in iliotibial band friction syndrome in runners: a randomized controlled trail British Journal of Sports Medicine 38:269-272

Hodges P, Richardson C 1996 Inefficient muscular stabilization of the limber spine associated with low back pain: A motor control evaluation of transverses abdominals. Spine 21:2640-2650

Hollingshead WH, Jenkins DB 1982 Functional Anatomy of the Limbs and back. Philidelphia: Saunders 265

Hyde SA, Scott CM, Goddard CM 1983 The myometer; the development of a clinical tool. Physiotherapy 69: 424-427

Khaund R, Flynn S.H, 2005 Iliotibial Band Syndrome: A Common Source of Knee Pain. American Family Physician 71:1545-1550

Maffiuletti N 2010 Assessment of hip and knee muscle function in orthopaedic practice and research. Journal of Bone Joint and Surgery of America 92:220-229

McClay I, Manal K 1999 Three-dimensional kinetic analysis of running: significance of secondary planes of motion. Med Sci of Sports Exerc 22:272-279

Miller R, Lowry J, Meardon S, Gillette J 2006 Lower extremity mechanics of iliotibial band syndrome during an exhaustive run. Gait \& Posture 26:407-413

Niemuth P, Johnson R, Myers M, Thiemann T 2005 Hip muscle weakness and overuse injuries in recreational runners. Clinical Journal of Sport Medicine 15: 14-21

Noehren B, Davis I, Hamill J 2007 ASB Clinical Biomechanics Award Winner 2006: Prospective study of the biomechanical factors associated with iliotibial band syndrome. Clinical Biomechanics 22:951-95

Scheffer C, Cloete T 2008 Benchmarking of a Full-Body Inertial Motion Capture System for Clinical Gait Analysis. Department of Mechanical and Mechatronic Engineering [dissertation], Stellenbosch University.
Taunton J, Ryan B, Clement B, Mckenzie C, Lloyd-Smith R, Zumbo D 2002 A retrospective case-control analysis of 2002 running injuries. British Journal of Sport Medicine 36:95-101 